Forum $2017 \cdot 32: 282$

DOI 10.1007/s12312-017-0295-x

Online publiziert: 28. Juli 2017

○) Springer Medizin Verlag GmbH 2017

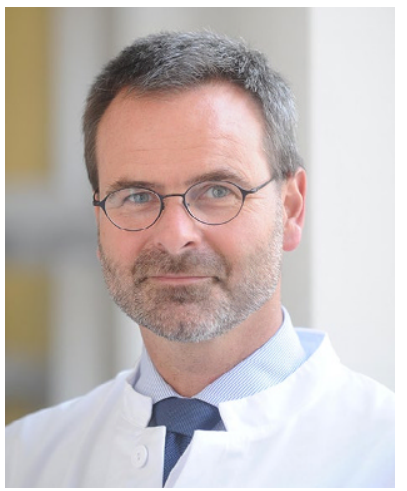

Bilanz ziehen ist von Zeit zu Zeit hilfreich. Der Abgleich zwischen Ist- und Sollzustand bewahrt davor, in Routine zu erstarren und schärft den Blick für offene Fragen und unerledigte Aufgaben. Die Deutsche Krebsgesellschaft nutzt üblicherweise das jährliche Delegiertentreffen im Sommer, um das Vorjahr Revue passieren zu lassen. Die gute Botschaft lautet: Unsere Bilanz im vergangenen Jahr kann sich sehen lassen. Unser Zertifizierungsprogramm besitzt Modellcharakter und erweist sich, ebenso wie das 2008 ins Leben gerufene Leitlinienprogramm Onkologie, als wichtiges Instrument der onkologischen Qualitätssicherung. Mittlerweile nimmt die DKG mit Unterstützung des Bundesministeriums für Gesundheit sogar an der Entwicklung verschiedener Qualitätssicherungs-Projekte der Europäischen Union teil. Unsere Arbeit und unser Engagement im Nationalen Krebsplan haben dazu geführt, dass die Stimme der DKG von der Politik gehört wird. Dabei hat sich die enge Zusammenarbeit mit der Deutschen Krebshilfe, die vor fünf Jahren durch einen Kooperationsvertrag besiegelt wurde, bewährt, auf dem politischen Parkett ebenso wie auf Projektebene. Auch mit anderen Fachgesellschaften befinden wir uns fortlaufend im Dialog, so zum

Peter Albers

Präsident der Deutschen Krebsgesellschaft e.V.

\title{
„Die DKG ist eine feste Größe in der Onkologie."
}

Beispiel mit der Deutschen Gesellschaft für Hämatologie und Onkologie bei Stellungnahmen oder Zertifizierungsthemen.

Bleibt die Frage, wie es insgesamt um die Krebsversorgung in unserem Land steht. Fakt ist: Dank besserer Therapien leben Patienten heute länger mit ihrem Krebs als noch vor einigen Jahren. Doch wie kommen innovative medizinische Maßnahmen schnell und sicher zu den Patienten? Kann sich unser System angesichts steigender Gesundheitsausgaben in Zukunft noch teure innovative Therapien und die dazugehörige Diagnostik leisten? Wie schaffen wir überall im Land gleiche Zugangsmöglichkeiten, unabhängig von Wohnort oder sozialem Status des Patienten? Die Chancengleichheit in der onkologischen Versorgung ist ein komplexes Problem, auf das dieses Heft näher eingeht.

\section{》) Vorgelegt: ein Papier zu "Wissen generierenden onkologi- schen Versorgung"}

Die DKG hat im Frühjahr 2017 gemeinsam mit Vertretern aus Politik, Ärzteschaft, Leistungsträgern und $\mathrm{Pa}$ tientenschaft ein Papier zur „Wissen generierenden onkologischen Versorgung “ vorgelegt. Die Arbeitsgruppe schlägt vor, dass der Zugang zu medizinischen Innovationen künftig über sogenannte translationale Tumorboards erfolgt, die bei der Anwendung eines neuen Verfahrens im Rahmen regionaler Versorgungsnetzwerke eng mit den behandelnden Ärzten kooperieren. Alle an der Behandlung Beteiligten verpflichten sich zur Dokumentation der Behandlungsdaten in klinischen Krebsregistern. Ergänzt werden soll dieses Netzwerk durch ein Lotsensystem, das dem Patienten hilft, sich wäh- rend des gesamten Krankheitsverlaufs im komplexen Gesundheitssystem zurechtzufinden. Das Modell ist die Antwort auf einige grundsätzliche Probleme in unserem Gesundheitswesen: Es würde solide Daten liefern, um den Nutzen von Innovationen im Vergleich zu etablierten Behandlungsstandards besser beurteilen $\mathrm{zu}$ können. Es ermöglicht eine höhere Patientensicherheit, weil die Betroffenen unter kontrollierten Bedingungen betreut würden. Es hilft den Patienten, die richtigen Anlaufstellen im System zu finden. Und bei Innovationen an der Schwelle zur Regelversorgung hätten wir am Ende bessere Argumente für oder gegen ihre Aufnahme in den GKV-Leistungskatalog.

Die Bundestagswahl 2017 steht vor der Tür. In ihrem Wahlprogramm verspricht die CDU, mehr in die Forschung gegen Krebs, Demenz und Alzheimer zu investieren; die SPD will sich dafür einsetzen, dass medizinische Neuerungen schnell und zu fairen Preisen vom ersten Tag an verfügbar sind. Parlamentarier beider Parteien waren an der Entstehung des Positionspapiers zur „Wissen generierenden onkologischen Versorgung " beteiligt. Wer auch immer in der nächsten Legislaturperiode für die Gesundheitspolitik verantwortlich sein wird - im Interesse der Patienten hoffe ich sehr, dass die Wissen generierende onkologische Versorgung aufgenommen und umgesetzt wird.

Ihr

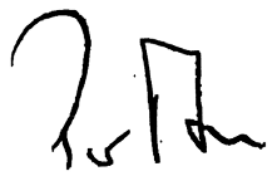

Ihr Peter Albers

Präsident der Deutschen Krebsgesellschaft 